Pacific Journal of Mathematics

THEORIES WITH A FINITE NUMBER OF MODELS IN AN 


\title{
THEORIES WITH A FINITE NUMBER OF MODELS IN AN UNCOUNTABLE POWER ARE CATEGORICAL
}

\author{
Alistair H. LACHLAN
}

In this paper are considered complete, countable, firstorder theories which have a finite number of models in some uncountable power. It is shown that any such theory is either $\omega$-categorical or $\omega_{1}$-categorical. This confirms a conjecture of W. K. Forrest.

Some of the background of this theorem is as follows. Shelah has shown that every non- $\omega$-stable theory has infinitely many models in every uncountable power. A proof of this will appear in Shelah [9]. Thus here we may confine our attention to $\omega$-stable theories. In [7] Shelah showed that a non- $\omega_{1}$-categorical, $\omega$-stable theory has $\geqq|\alpha+1|$ models in power $\boldsymbol{\aleph}_{\alpha}$. Another proof of the same result was published by Rosenthal [6]. Thus the only uncountable cardinals $\kappa$ in which a complete countable theory can have a finite number of, and at least two, models are the $\aleph_{n}$ for $1<n<\omega$. The methods used in this paper come from Baldwin and Lachlan [1] and from several works by Shelah.

1. Preliminaries. Most of our notation is patterned on [8]. An additional notation used here is $\varphi(M, \bar{a})$ for the set of solutions of the formula $\varphi(x, \bar{a})$ in the structure $M$. Also, if $A \subset|M|$ by $\operatorname{cl}(A)$ we mean the union of all finite subsets of $M$ definable using parameters from $A$. We call cl $(A)$ the algebraic closure or simply closure of $A$. The reader will require some knowledge of the theory of strongly minimal sets such as may be found in [1].

Let $M$ be a structure. A formula $\varphi(x, \bar{a}), \bar{a} \in M$, is called strongly minimal if it has an infinite solution set in $M$ and for every elementary extension $M^{\prime}$ of $M$, formula $\psi(x, \bar{y})$, and $\bar{b} \in M^{\prime}$, one of the formulas $\varphi(x, \bar{a}) \wedge \psi(x, \bar{b})$ and $\varphi(x, \bar{a}) \wedge \neg \psi(x, \bar{b})$ has a finite solution set in $M^{\prime}$.

A formula $\varphi(x, \bar{a}), \bar{a} \in M$, is called $\omega$-categorical if its solution set is infinite and for every $n<\omega$ there are at most a finite number of $n$-types over $\mathrm{Rng} \bar{a}$ realized by $n$-tuples in the solution set of $\varphi(x, \bar{a})$.

If $\operatorname{Th}(M)$ is superstable, $\varphi(x, \bar{a})$ is $\omega$-categorical in $M$, and $A \subset|M|$ is finite, then $\varphi(x, \bar{a})$ is also $\omega$-categorical in $M^{\prime}$ the expansion of $M$ obtained by adjoining names for the members of $A$. This follows from the following definability result: Let $\operatorname{Th}(M)$ be 
superstable, $\bar{b} \in M$, and $A$ be a subset of $|M|$ definable without parameters, then there exists $\bar{c} \in A$ such that if $\varphi(z, \bar{y})$ is any formula with $\operatorname{lh}(\bar{b})=\operatorname{lh}(\bar{y})$ then some equivalence relation $\mathscr{C}$ on $M$ is definable from $\bar{c}$ such that $A / \mathscr{E}$ is finite and $A \cap \varphi(M, \bar{b})$ is $\mathscr{E}$-closed. We shall not prove this here. A stronger theorem in which $A$ need not be definable will appear in [9]. Besides, if one looks closely at the proof of our main theorem it is apparent that we can avoid using the fact that an $\omega$-categorical formula remains $\omega$-categorical when a finite number of elements are named.

In passing we note that if $\varphi(x, \bar{a})$ is $\omega$-categorical in $M$ and $\operatorname{Th}(M)$ is stable it may not be the case that $\varphi(x, \bar{a})$ is $\omega$-categorical in every inessential expansion of $M$. For example, consider the case in which the language consists of binary relations $R_{0}, R_{1}, \ldots$ Let $|M|=\omega \cup{ }^{\omega} \omega$ and for $i<\omega$ let $R_{i}^{M}(a, b)$ hold just if $a \in{ }^{\omega} \omega$ and $a(i)=b$. Then $\mathrm{Th}(M)$ is stable and $\exists y R_{0}(y, x)$ is $\omega$-categorical in $M$ but not in $M^{\prime}$ obtained by adjoining a name for a member of ${ }^{\omega} \omega$ which has infinite range.

If $\varphi(x, \bar{a})$ is an s.m. formula in $M$, its dimension in $M$ denoted $\operatorname{dim}(\varphi,(x, \bar{a}), M)$ is the cardinality of a minimal set $A \subset \varphi(M, \bar{a})$ such that $\varphi(M, \bar{a}) \subset \operatorname{cl}(A \cup \operatorname{Rng} \bar{a})$. From the theory developed in [1] the choice of $A$ makes no difference. Likewise we talk of independent sets of solutions of $\varphi(x, \bar{a})$, of a basis for $\varphi(x, \bar{a})$ in a particular model and so on. We adjoin names for the members of $\bar{a}$ and then use the definitions already made for formulas without parameters. We call $\varphi(x, \bar{b})$ a copy of $\varphi(x, \bar{a})$ if $\bar{b}$ realizes the same type as $\bar{a}$.

All theories considered are assumed to be complete and countable.

2. Stability and strongly minimal formulas. In this section are presented some lemmas which will be useful for the proof of the main theorem. Most of the lemmas have been proved in a more general context than is necessary for their application in this paper. This is because of our continuing interest in the theory of stability in general and strongly minimal formulas in particular.

Lemma 1. Let $A$ be a subset of a model of a stable theory $T$. There exists a model $M$ of $T$ such that $A \subset|M|$ and further: for each $a \in M$ and formula $\varphi(x, \bar{y})$ there exist $\bar{c} \in A$ and $\theta(x, \bar{z})$ such that $\vDash \theta(a, \bar{c})$ and for all $\bar{b} \in A$

$$
\{\vDash \varphi(a, \bar{b})\} \Longrightarrow\{\vDash \forall x[\theta(x, \bar{c}) \longrightarrow \varphi(x, \bar{b})]\} \text {. }
$$

This was discovered independently by Shelah and the author. Shelah was the first to formulate it explicitly [8, p. 275]. A proof 
of it can be extracted from the second half of the proof of the model extension theorem in [2].

Lemma 2. Let $\mathrm{Th}(M)$ be $\omega$-stable, $N$ be an elementary extension of $M, \bar{a} \in N, \varphi(x, \bar{a})$ and $\psi(x)$ be formulas. Further, suppose $\varphi(x, \bar{a})$ implies $\psi(x)$ and that $\varphi(N, \bar{a}) \cap|M|=\varnothing$ then $\varphi(x, \bar{a})$ has rank less than that of $\psi(x)$.

This can be proved using the same technique as in the proof of Lemma 7 of [2]. In the application we make of this lemma $M$ is the prime model and $\varphi(N, \bar{a}) \cap|M|=\varnothing$ is satisfied because $\varphi(x, \bar{a})$ has no solution realizing an isolated type over $\varnothing$.

A formula $\varphi(x)$ is said to have the Vaught property in a theory $T$ if there are models $M$ and $N$ of $T$ such that $N$ is a proper elementary extension of $M, \varphi(M)=\varphi(N)$, and $|\varphi(M)| \geqq \omega$.

Lemma 3. Let $T$ be a superstable theory and $\varphi(x)$ be a strongly minimal formula which has the Vaught property. Suppose further that $\varphi(x)$ has infinitely many algebraic solutions. Then there exist arbitrarily large models in which the dimension of $\varphi(x)$ is finite.

Proof. Since $\varphi(x)$ has the Vaught property and $T$ is stable there exists an arbitrarily large model $M$ of $T$ with $|\varphi(M)|=\omega$. This is immediate from the extension theorem of [2]. By Theorem 3.1 of [7] if such $M$ is sufficiently large it contains a subset $I$ of power $\omega$ indiscernible over $\varphi(M)$.

We shall first establish that $\operatorname{cl}(I) \cap \varphi(M)$ has finite dimension. For proof by contradiction suppose the contrary. From Theorem 6.13(A) of [8] it is clear that if $I_{0} \subset I$ is finite then $\operatorname{cl}\left(I_{0}\right) \cap \varphi(M)$ has finite dimension. Also from the indiscernibility of $I$ over $\varphi(M)$, $\operatorname{cl}\left(I_{0}\right) \cap \varphi(M)$ depends only on $\left|I_{0}\right|$. Let $e_{0}, e_{1}, \cdots$ be an enumeration of a basis of $\mathrm{cl}(I) \cap \varphi(M)$, and $a_{0}, a_{1}, \cdots$ be an enumeration of $I$. For each $n<\omega$ let $m(n)$ be the least number such that $\left\{e_{0}, e_{1}, \cdots, e_{n}\right\} \subset$ cl $\left\{a_{0}, a_{1}, \cdots, a_{m(n)}\right\}$. It is clear that $m(n)$ is nondecreasing and unbounded as $n$ increases. Since it will simplify the notation without materially affecting the argument we shall suppose that $m(n)=n$. Let $\theta_{n}\left(x, a_{0}, a_{1}, \cdots, a_{n}\right)$ be a formula with only a finite number of solutions one of which is $e_{n}$. Let $\psi_{n}(x)$ denote the formula:

$$
\theta_{0}\left(e_{0}, x\right) \wedge \theta_{1}\left(e_{1}, a_{0}, x\right) \wedge \cdots \wedge \theta_{n}\left(e_{n}, a_{0}, a_{1}, \cdots, a_{n-1}, x\right) .
$$

Notice that $e_{n+1}, e_{n+2}, \cdots$ all realize the same type over $\left\{e_{i}: i \leqq n\right\} \cup$ $\left\{a_{i}: i \leqq n\right\}$. By suitable choice of $\theta_{n+1}$ for any sufficiently large finite $w \subset \omega$

$$
\vDash \neg \exists x \wedge\left\{\theta_{n+1}\left(e_{m}, a_{0}, \cdots, a_{n}, x\right): m \in w\right\} .
$$


Thus the formulas $\psi_{n}(x) \wedge \theta_{n+1}\left(e_{m}, a_{0}, \cdots, a_{n}, x\right)$ for $m=n+1, n+2, \cdots$ which all have the same degree in the sense of Shelah [8], and the first of which is $\psi_{n+1}(x)$, witness that Deg $\psi_{n+1}<\operatorname{Deg} \psi_{n}$. This contradicts the superstability of $T$ by Corollary $6.10(\mathrm{~A})$ of [8]. Thus cl $(I) \cap \varphi(M)$ does indeed have finite dimension.

To complete the proof of the theorem extend $I$ to a set $I^{\prime}$, indiscernible over $\varphi(M)$, of any desired cardinality and apply Lemma 1 to $\mathrm{cl}\left(I^{\prime}\right)$.

LEMMA 4. Let $T$ be a complete theory and $\varphi(x)$ be a strongly minimal formula which does not have the Vaught property, then $T$ is $\omega_{1}$-categorical.

Proof. From [1], see proof of Lemma 8, p. 83, the present lemma is obvious when $T$ is $\omega$-stable. Suppose therefore that $T$ is not $\omega$-stable. Form an increasing sequence $M(0), M(1), \cdots$ of countable models of $T$ such that $|S(M(0))|>\omega$ and such that for all $i$ $\varphi(M(i+1)) \neq \varphi(M(i))$. Let $M=\bigcup\{M(i): i<\omega\}$. Let $\left\langle\varphi_{i}\left(x, \bar{a}^{i}\right): i<\omega\right\rangle$ be an enumeration of all formulas having only $x$ free and parameters from $M$, similarly let $\left\langle\psi_{i}\left(x, y, \bar{b}^{i}\right): i\langle\omega\rangle\right.$ be an enumeration of all formulas having at most $x, y$ free. We construct a sequence of triples $\left\langle\left\langle\theta_{i}\left(x, \bar{c}^{i}\right), n_{i}, a_{i}\right\rangle: i\langle\omega\rangle\right.$ such that for all $i$ the following five conditions hold:

(i ) $n_{i}<n_{i+1}<\omega, \bar{c}^{i} \in M\left(n_{i}\right)$, and $a_{i} \in \varphi\left(M\left(n_{i+1}\right)\right)$

(ii) $\theta_{i+1}\left(x, \bar{c}^{i+1}\right)$ implies $\theta_{i}\left(x, \bar{c}^{i}\right)$

(iii) $\theta_{i}\left(x, \bar{c}^{i}\right)$ implies one of the formulas $\varphi_{i}\left(x, \bar{a}^{i}\right)$ and $\neg \varphi_{i}\left(x, \bar{a}^{i}\right)$

(iv) $\left|S\left(M\left(n_{i}\right)\right) \cap\left\{p: \theta_{i}\left(x, \bar{c}^{i}\right) \in p\right\}\right|>\omega$

(v) if $\vDash \exists x \exists y\left(\theta_{i}\left(x, \bar{c}^{i}\right) \wedge \psi_{i}\left(x, y, \bar{b}^{i}\right) \wedge \varphi(y)\right)$, then $\theta_{i}\left(x, \bar{c}^{i}\right) \wedge \psi_{i}(x$, $\left.a_{i}, \bar{b}^{i}\right)$ is consistent with $>\omega$ members of $S\left(M\left(n_{i}\right)\right)$ and $\theta_{i+1}\left(x, \bar{c}^{i+1}\right)$ implies $\psi_{i}\left(x, a_{i}, \bar{b}^{i}\right)$.

The only part of the construction which is not straightforward is the choice of $a_{i}$. Suppose the triples prior to $\left\langle\theta_{i}\left(x, \bar{c}^{i}\right), n_{i}, a_{i}\right\rangle$ have all been suitably chosen, and that $\theta_{i}\left(x, \bar{c}^{i}\right)$ and $n_{i}$ have been chosen so that (i)-(iv) are satisfied. Call a formula $\chi(x, \bar{a})$ good if its parameters are in $M\left(n_{i}\right)$, it implies $\theta_{i}\left(x, \bar{c}^{i}\right)$, and

$$
\left|S\left(M\left(n_{i}\right)\right) \cap\{p: \chi(x, \bar{a}) \in p\}\right|>\omega .
$$

Notice that if $\chi(x, \bar{a})$ is good, then there are disjoint good formulas $\chi_{0}\left(x, \bar{a}^{0}\right)$ and $\chi_{1}\left(x, \bar{a}^{1}\right)$ such that $\chi(x, \bar{a})$ is equivalent to $\chi_{0}\left(x, \bar{a}^{0}\right) \vee$ $\chi_{1}\left(x, \bar{a}^{1}\right)$. Suppose there is a good formula $\chi(x, \bar{a})$ such that $\vDash \neg \exists x \exists y\left(\chi(x, \bar{a}) \wedge \psi_{i}\left(x, y, \bar{b}^{i}\right) \wedge \varphi(y)\right)$. Then we can satisfy (v) by replacing $\theta_{i}\left(x, \bar{c}^{i}\right)$ by $\chi(x, \bar{a})$. The choice of $a_{i}$ is immaterial. If for some good $\chi(x, \bar{a})$ the solution set of $\exists x\left(\chi(x, \bar{a}) \wedge \psi_{i}\left(x, y, \bar{b}^{i}\right) \wedge \varphi(y)\right)$ 
is nonempty but finite, then for some $b \in \varphi\left(M\left(n_{i}\right)\right), \chi(x, \bar{a}) \wedge \psi_{i}\left(x, b, \bar{b}^{i}\right)$ is good. Thus to satisfy (v) in this case we just take $a_{i}=b$. Otherwise let $a_{i} \in \varphi\left(M\left(n_{i}+1\right)\right)-\varphi\left(M\left(n_{i}\right)\right)$. For each good $\chi(x, \bar{a})$ we now have from the strong minimality of $\varphi(x)$

$$
\vDash \exists x\left(\chi(x, \bar{a}) \wedge \psi_{i}\left(x, a_{i}, \bar{b}^{i}\right) \wedge \varphi\left(a_{i}\right)\right),
$$

whence this choice of $a_{i}$ will again satisfy (v).

Let $a$ realize the type over $M$ generated by the formulas $\theta_{i}\left(x, \bar{c}^{i}\right)$. By the omitting types theorem there is a model $M^{\prime} \supset|M| \cup\{a\}$ such that $\varphi\left(M^{\prime}\right)=\varphi(M)$. Thus $\varphi(x)$ does have the Vaught property.

Lemma 5. Let $T$ be a stable theory for which $\varphi(x)$ and $\psi(x)$ are s.m. formulas. Further suppose that each of these two formulas has infinitely many algebraic solutions. Then either in every model of $T \varphi(x)$ and $\psi(x)$ have the same dimension, or each model $M$ of $T$ has an elementary extension $M^{\prime}$ such that $\psi(x)$ has the same solution set in $M^{\prime}$ as in $M$ while $\varphi\left(M^{\prime}\right)=\operatorname{cl}(\varphi(M) \cup\{b\}, M)$ for some $b$ in $\left|M^{\prime}\right|-|M|$. The former case holds if and only if there is a formula $\chi(x, y)$ such that the following are valid in $T$ :

$$
\chi(x, y) \longrightarrow \varphi(x) \wedge \psi(y), \exists^{<\omega} y(\chi(x, y) \wedge \psi(y))
$$

and $\exists^{\geqq \omega} y \exists x \chi(x, y)$.

Proof. To prove the first part of the lemma we attempt to construct $M^{\prime}$. Let $A=|M| \cup\{b\}$ where $b$ is a solution of $\varphi(x)$ not in $M$. Apply Lemma 1 to $A$ to form $M^{\prime}$.

Suppose that $\psi(x)$ has a solution $a$ in $\left|M^{\prime}\right|-|M|$. Taking $\psi(x) \wedge x \neq y_{0}$ as $\varphi(x, \bar{y})$ in the statement of Lemma 1 we find a formula $\theta(x, y, \bar{z})$ and $\bar{c} \in M$ such that $\vDash \theta(a, b, \bar{c})$ and $\theta(x, b, \bar{c})$ has no solution in $M$. We can suppose that $\theta(x, y, \bar{z})$ implies $\psi(x) \wedge \varphi(y)$. Clearly $\vDash \exists x \theta(x, b, \bar{c}), \quad b$ is not algebraic over $|M|$, and $\phi$ is s.m. It follows that for some $k<\omega, \vDash \exists^{k} y \neg \exists x \theta(x, y, \bar{c})$. Similarly, $\vDash \exists^{l} x \neg \exists y \theta(x, y, \bar{c})$ for some $l<\omega$. Now all the solutions of $\theta(x, b, \bar{c})$ satisfy $\psi(x)$ and none are in $M$, hence there are at most a finite number, say $m$. We may suppose that

$$
\vDash \forall y\left(\exists y \theta(x, y, \bar{c}) \longrightarrow \exists^{m} x \theta(x, y, \bar{c})\right) .
$$

Since $a \notin M, \theta(a, y, \bar{c})$ cannot have a solution for $y$ in $M$, whence $\theta(a, y, \bar{c})$ has only a finite number since $\varphi(x)$ is s.m. Hence we may suppose that for some $n<\omega$

$$
\vDash \forall x\left(\exists x \theta(x, y, \bar{c}) \longrightarrow \exists^{m} y \theta(x, y, \bar{c})\right) .
$$

From Theorem 3.1 (A) ([8], p. 306) we can find a formula $\theta\left(x, y, \bar{z}^{\prime}\right)$ 
and parameters $\bar{c}^{\prime}$ in $\varphi(M) \cup \psi(M)$ such that

$$
\vDash \exists^{k} y \neg \exists x \theta^{\prime}\left(x, y, \bar{c}^{\prime}\right) \wedge \exists^{l} x \neg \exists y \theta^{\prime}\left(x, y, \bar{c}^{\prime}\right) \wedge
$$

$\forall x \forall y\left(\theta^{\prime}\left(x, y, \bar{c}^{\prime}\right) \rightarrow \psi(x) \wedge \varphi(y)\right) \wedge \forall y\left(\exists x \theta^{\prime}\left(x, y, \bar{c}^{\prime}\right) \rightarrow \exists^{m} x \theta^{\prime}\left(x, y, \bar{c}^{\prime}\right)\right)$

$$
\Lambda \forall x\left(\exists y \theta^{\prime}\left(x, y, \bar{c}^{\prime}\right) \longrightarrow \exists^{n} y \theta^{\prime}\left(x, y, \bar{c}^{\prime}\right)\right) .
$$

To see this we apply the theorem of Shelah just cited taking $A$ to be $\varphi(M) \cup \psi(M), \varphi(\bar{x}, \bar{y})$ to be $\theta(x, y, \bar{z})$ where $\bar{z}$ plays the role of $\bar{x}$ and $\langle x, y\rangle$ the role of $\bar{y}$, and $p$ to be the $\theta$-type of $\bar{c}$.

Since both $\varphi(x)$ and $\psi(x)$ have infinitely many algebraic solutions there is a formula $\tau\left(\bar{z}^{\prime}\right)$ with a finite nonempty set of solutions any of which will serve as $\bar{c}^{\prime}$. The parameterless formula $\exists \bar{z}^{\prime}\left(\tau\left(\bar{z}^{\prime}\right) \wedge\right.$ $\left.\theta^{\prime}\left(x, y, \bar{z}^{\prime}\right)\right)$ defines a correspondence between the solution set of $\psi(x)$ and the solution set of $\varphi(x)$. The correspondence is finite-one in both directions, all but a finite number of solutions of $\psi(x)$ have mates in $\varphi(x)$ and vice versa. In a given model of $T$ let $X$ be a basis of $\psi(x)$. Form $Y$ by choosing for each $e$ in $X$ one of its mates under the correspondence to be a member of $Y$. It is easily seen that $Y$ is a basis of $\varphi(x)$ and that its cardinality is necessarily the same as that of $X$. This shows that the lemma is true when $\psi(x)$ has a solution in $\left|M^{\prime}\right|-|M|$.

There remains only the task of showing that $\varphi\left(M^{\prime}\right)$ is $\operatorname{cl}(\varphi(M) \cup$ $\left.\{b\}, M^{\prime}\right)$. This is immediate from the application of Lemma 1 by which $M^{\prime}$ was formed. We just take $\varphi(x, \bar{y})$ in the statement of the lemma to be $\varphi(x) \wedge x \neq y_{0}$.

The second part of the lemma is immediate from the discussion above. We can take $\chi(x, y)$ to be $\exists \bar{z}^{\prime}\left(\tau\left(\bar{z}^{\prime}\right) \wedge \theta^{\prime}\left(x, y, \bar{z}^{\prime}\right)\right)$.

Let $\varphi(x, \bar{a})$ and $\psi(x, \bar{b})$ be two s.m. formulas in a model $M$ of T. Adjoin names for the members of $\operatorname{Rng}\left(\bar{a}^{\cap} \bar{b}\right)$ and then names for suitable members of $\varphi(M, \bar{a})$ and $\psi(M, \bar{b})$ in such a way that if $A \subset|M|$ is the set named, then $|A| \leqq \omega, A$ is prime over Rng $\left(\bar{a}^{\cap} \bar{b}\right)$, and

$$
|\operatorname{cl}(A) \cap \varphi(M, \bar{a})|=\omega=|\operatorname{cl}(A) \cap \psi(M, \bar{b})| .
$$

Let $M^{\prime}$ be the resulting expansion of $M$, and $T^{\prime}$ be the corresponding extension of $T$. We say that $\varphi(x, \bar{a})$ and $\psi(x, \bar{b})$ are linked if and only if in every model of $T^{\prime}$ they have the same dimension. Note that this definition is independent of the way in which $M^{\prime}$ is formed.

Clearly, if $\varphi(x, \bar{a})$ and $\psi(x, \bar{b})$ are linked then in any model of $T$ containing $\bar{a}$ and $\bar{b}, \varphi(x, \bar{a})$ and $\psi(x, \bar{b})$ have the same dimension modulo $\omega$. The following may easily be deduced from the last lemma. Since it is useful we state it explicitly and leave the proof to the reader: 
LEMMA 6. Let $\varphi(x)$ and $\psi(x)$ be s.m. formulas of a stable theory $T$. Let $T^{\prime}$ be an inessential extension of $T$. If $\varphi(x)$ and $\psi(x)$ are linked in $T^{\prime}$ they are linked in $T$.

LEMMA 7. Let $T$ be stable and $\varphi_{0}(x), \cdots, \varphi_{n-1}(x)$ be s.m. formulas. For each $\kappa>\omega$ there exists a model $M$ of $T$ of power $\kappa$ such that if $\varphi(x, \bar{a})$ is any s.m. formula of $M$ then $|\varphi(M, \bar{a})|=\kappa$ if and only if $\varphi(x, \bar{a})$ is linked to one of $\varphi_{0}(x), \cdots, \varphi_{n-1}(x)$.

Proof. Without loss of generality we may assume that each $\varphi_{i}(x)$ contains infinitely many algebraic elements. Choose $A_{0}, \cdots, A_{n-1}$ such that for each $i<n, A_{i}$ is an independent set of solutions of $\varphi_{i}(x)$ and $\left|A_{i}\right|=\kappa$. We now form a model $M$ of $T$ including $A=A_{0} \cup \cdots \cup A_{n-1}$ using the same technique which yields Lemma 1. In fact $|M|=\left\{b_{i}: i<\kappa\right\}$ where $b_{0}, b_{1}, \cdots$ are chosen in turn. To make $M$ a model we arrange that if a formula $\varphi\left(\bar{a}, \bar{b}_{i_{0}}, \cdots, b_{i_{k-1}}, x\right)$ is consistent where $\bar{a} \in A$ then there exists $j<\kappa$ such that $\vDash \varphi\left(\bar{a}, b_{i_{0}}\right.$, $\left.\cdots, b_{i_{k-1}}, b_{j}\right)$. For the rest we simply choose $b_{i}$ in such a way that for any formula $\varphi(x, \bar{y})$ there exists a formula $\theta(x, \bar{z})$ and $\bar{c} \in A \cup$ $\left\{b_{j}: j<i\right\}$ such that $\vDash \theta\left(b_{i}, \bar{c}\right)$ and such that for any $\bar{b} \in A \cup\left\{b_{j}: j<i\right\}$ if $\vDash \varphi\left(b_{i}, \bar{b}\right)$ then $\vDash \forall x(\theta(x, \bar{c}) \rightarrow \varphi(x, \bar{b}))$. Using the stability and countability of $T$ the model $M$ can easily be formed according to the above prescription.

Let $\varphi(x, \bar{a})$ be an s.m. formula in $M$ whose parameters are from $\left\{b_{j}: j<i\right\}$ where $\omega<i<\kappa$. Let $C=\operatorname{cl}\left(A \cup\left\{b_{j}: j<i\right\}\right)$ then without loss we may suppose that $\varphi(M, \bar{a}) \cap C$ is infinite. Suppose $|\varphi(M, \bar{a}) \cap C|>|i|$. Then there exists $j<n$ and a formula $\chi\left(a_{j}, x\right)=$ $\psi\left(\bar{b}, \bar{a}^{0}, \bar{a}^{1}, \cdots, \bar{a}^{j}, a_{j}, x\right)$ such that $\bar{b} \in\left\{b_{j}: j<i\right\}, \bar{b}$ extends $\bar{a}, \bar{a}^{k} \in A_{k}$ for each $k \leqq j$,

$$
a_{j} \in A_{j}-\operatorname{cl}\left(\operatorname{Rng} \bar{b} \cup \operatorname{Rng} \bar{a}^{0} \cup \cdots \cup \operatorname{Rng} \bar{a}^{j}\right)
$$

and $\chi\left(a_{j}, x\right)$ has a solution in

$$
\varphi(M, \bar{a})-\operatorname{cl}\left(\operatorname{Rng} \bar{b} \cup \operatorname{Rng} \bar{a}^{0} \cup \cdots \cup \operatorname{Rng} \bar{a}^{j}\right) .
$$

The formula $\chi(y, x) \wedge \varphi(x, \bar{a}) \wedge \varphi_{j}(y)$ defines a correspondence between the solutions of $\varphi(x, \bar{a})$ and those of $\varphi_{j}(x)$, whence in every elementary extension of $M \varphi(x, \bar{a})$ and $\varphi_{j}(x)$, have the same number of solutions. From Lemma 5 any model containing two unlinked s.m. formulas has an elementary extension in which those formulas have different numbers of solutions. Thus in this case $\varphi(x, \bar{a})$ and $\varphi_{j}(x)$ are linked. Suppose $|\varphi(M, \bar{a}) \cap C| \leqq|i|$ then it is easy to show by induction on $j$ that if $i \leqq j \leqq \kappa, \bar{b} \in A \cup\left\{b_{k}: k<j\right\}$ and $\psi(x, \bar{b})$ has a solution in $\varphi(x, \bar{a})$, then it has a solution in $\varphi(M, \bar{a}) \cap C$. It follows that in 
this case $\varphi(M, \bar{a})=\varphi(M, \bar{a}) \cap C$ which has power $<\kappa$. This completes the proof of the lemma.

LEMMA 8. Let $\varphi_{0}\left(x, \bar{a}^{0}\right)$ and $\varphi_{1}\left(x, \bar{a}^{1}\right)$ be linked s.m. formulas in a model $M$ of a stable $T$. Let $\operatorname{dim}\left(\varphi_{i}\left(x, \bar{a}^{i}\right), M\right)=n_{i}<\omega$ for $i<2$. Then for any model $N$ of $T$ containing $\bar{a}^{0}$ and $\bar{a}^{1}$

$$
\operatorname{dim}\left(\varphi_{0}\left(x, \bar{a}^{0}\right), N\right)-\operatorname{dim}\left(\varphi_{1}\left(x, \bar{a}^{1}\right), N\right)=n_{0}-n_{1}
$$

provided that one of the dimensions on the left is finite.

Proof. Suppose the hypotheses are satisfied. For $i<2$ choose $\bar{b}^{j}$ such that Rng $\bar{b}^{i}$ is an independent set of solutions of $\varphi_{i}\left(x, \bar{a}^{i}\right)$, and $\varphi_{i}\left(x, \bar{a}^{i}\right)$ has infinitely many solutions in $\operatorname{cl}\left(\bar{a}^{i \cap} \bar{b}^{i}\right)$. Further make these choices so that $\bar{b}^{0} \cap \bar{b}^{1}$ is prime over $\bar{a}^{0} \bar{a}^{1}$. Within this restriction let $\bar{b}^{0}$ and $\bar{b}^{1}$ both be of maximal length. Let $m_{i}=\operatorname{lh}\left(\bar{b}_{i}\right)$ for $i<2$. Let $T^{\prime}$ be formed by adjoining names for the members of $\bar{a}^{0}, \bar{a}^{1}, \bar{b}^{0}$, and $\bar{b}^{1}$. Let $N$ be an arbitrary model of $T$ containing $\bar{a}^{0}$ and $\bar{a}^{1}$ and $N^{\prime}$ be an expansion of $N$ to a model of $T^{\prime}$. Let $\bar{c}^{0}$ and $\bar{c}^{1}$ correspond to $\bar{b}^{0}$ and $\bar{b}^{1}$ respectively under the expansion of $N$ to $N^{\prime}$. Let $\bar{c}^{0 \cap} \bar{d}$ be a basis for $\varphi_{0}\left(x, \bar{a}^{0}\right)$ in $N$. We claim that $\bar{d}$ is a basis for $\varphi_{0}\left(x, \bar{a}^{0}\right)$ in $N^{\prime}$. If not, then there exist $j<\omega$ and a formula $\psi\left(\bar{y}^{0}, \bar{y}^{1}, \bar{y}^{2}, \bar{y}^{3}, \bar{x}\right)$ such that

$$
\vDash \psi\left(\bar{a}^{0}, \bar{a}^{1}, \bar{c}^{0}, \bar{c}^{1}, d_{0}, \cdots, d_{j}\right) \wedge \exists^{\leqslant m} z \psi\left(\bar{a}^{0}, \bar{a}^{1}, \bar{c}^{0}, \bar{c}^{1}, \bar{d}_{0}, \cdots, \bar{d}_{j-1}, z\right)
$$

where $d_{0}, \cdots, d_{j}$ are distinct members of $\bar{d}$. Abbreviate $\psi\left(\bar{a}^{0}, \bar{a}^{1}, \bar{c}^{0}\right.$, $\left.\bar{c}^{1}, \bar{x}\right) \wedge \Lambda_{i \leqq j} \varphi_{0}\left(x_{i}, \bar{a}^{0}\right)$ to $\chi(\bar{x})$. From Theorem 3.1(A) [8, p. 306] the relation on $\varphi_{0}\left(N, \bar{a}^{0}\right)$ defined by $\chi(\bar{x})$ can be defined using only parameters from $\varphi_{0}\left(N, \bar{a}_{0}\right)$. Further since there is a model $N^{0}$ of $T$ such that $\bar{a}^{0}, \bar{a}^{1}, \bar{c}^{0}, \bar{c}^{1} \in N^{0}$ and $\varphi_{0}\left(N^{0}, \bar{a}^{0}\right) \subset \operatorname{cl}\left(\operatorname{Rng} \bar{a}^{0 \cap} \bar{c}^{0} \cap \bar{c}^{1}\right)$, it follows that there is a formula equivalent to $\chi(\bar{x})$ containing only parameters from $\varphi_{0}\left(N, \bar{a}^{0}\right)$ which are algebraic over $\bar{a}^{0 \cap} \bar{c}^{0}$. But then we should have

$$
\vDash \theta\left(d_{0}, \cdots, d_{j}\right) \wedge \exists^{\leqq n} z \theta\left(d_{0}, \cdots, d_{j-1}, z\right)
$$

for some $n<\omega$ and $\theta(\bar{x})$ containing only $\bar{a}^{0}$ and $\bar{c}^{0}$ as parameters. This contradicts the choice of $\bar{d}$. We have shown that

$$
\operatorname{dim}\left(\varphi_{0}\left(x, \bar{a}^{0}\right), N\right)=\operatorname{dim}\left(\varphi_{0}\left(x, \bar{a}^{0}\right), N^{\prime}\right)+m_{0}
$$

provided that the lefthand side is finite. From Lemma 5

$$
\operatorname{dim}\left(\varphi_{0}\left(x, \bar{a}^{0}\right), N^{\prime}\right)=\operatorname{dim}\left(\varphi_{1}\left(x, \bar{a}^{1}\right), N^{\prime}\right) .
$$

It follows that $\operatorname{dim}\left(\varphi_{0}\left(x, \bar{a}^{1}\right), N\right)$ is finite, and hence in exactly the same way as for $\varphi_{0}\left(x, \bar{a}^{0}\right)$ 


$$
\operatorname{dim}\left(\varphi_{1}\left(x, \bar{a}^{1}\right), N\right)=\operatorname{dim}\left(\varphi_{1}\left(x, \bar{a}^{1}\right), N^{\prime}\right)+m_{1} .
$$

This is enough to prove the lemma.

For the next lemma we are indebted to Shelah who pointed it out to the author in the course of conversation.

LEMMA 9. Let $\varphi(x)$ be a formula without parameters, $M_{0}$ and $M_{1}$ be models of an $\omega$-stable theory, and $F: \varphi\left(M_{0}\right) \rightarrow \varphi\left(M_{1}\right)$ be an elementary onto map. There is a model $M$ and elementary embeddings $F_{0}, F_{1}$ of $M_{0}, M_{1}$ into $M$ respectively such that

$$
F_{0}\left(\varphi\left(M_{0}\right)\right)=F_{1}\left(\varphi\left(M_{1}\right)\right)=\varphi(M) \text {. }
$$

Proof. We may suppose that $F$ is the identity on $\varphi\left(M_{0}\right)$, that $N$ is an elementary submodel of both $M_{0}$ and $M_{1}$ prime over $\varphi\left(M_{0}\right)$, and that $\left(\left|M_{0}\right|-|N|\right) \cap\left(\left|M_{1}\right|-|N|\right)=\varnothing$. We define a theory $T^{*}$ for the language obtained by adjoining names for all the members of $\left|M_{0}\right| \cup\left|M_{1}\right|$ as follows. Let $\theta(\bar{x}, \bar{y})$ be a formula of $T$ and $\bar{a}$ be a tuple in $\left|M_{1}\right|-|N|$ such that $l h(\bar{x})=l h(\bar{a})$. From Shelah [8] Theorem 3.1 (A) there is a formula $\psi_{\theta, \bar{a}}(\bar{y})$ with parameters from $N$ such that $\vDash \theta(\bar{a}, \bar{b}) \leftrightarrow \psi_{\theta, \bar{a}}(\bar{b})$ for all $\bar{b}$ in $N$. Let $T^{*}$ have as nonlogical axioms all sentences $\theta(\bar{a}, \bar{b})$ where $\bar{a} \in\left|M_{1}\right|-|N|, \bar{b} \in M_{0}$, and $\vDash \psi_{\theta, \bar{a}}(\bar{b})$. It is easy to see that $T^{*}$ is consistent, complete, and extends $T h\left(M_{0},\left|M_{0}\right|\right) \cup T h\left(M_{1},\left|M_{1}\right|\right)$.

A prime model $M^{*}$ of $T^{*}$ exists because $T$ is $\omega$-stable. The reduct of $M^{*}$ to the language of $T$ is the desired model $M$. One has to verify that if $\chi$ is a formula of $T$ such that $\exists z(\chi(\bar{a}, \bar{b}, z) \wedge$ $\varphi(z))$ is a theorem of $T^{*}$, where $\bar{a} \in\left|M_{1}\right|-|N|$ and $\bar{b} \in M_{0}$, then $\chi(\bar{a}, \bar{b}, c)$ is a theorem of $T^{*}$ for some $c \in \varphi(N)$. Using the theorem of Shelah quoted above let $\chi_{0}(\bar{y})$ and $\chi_{1}(\bar{y}, z)$ be formulas with parameters from $N$ such that for all $\bar{b}^{\prime}, c^{\prime}$ in $N \vDash \exists z\left(\chi\left(\bar{a}, \bar{b}^{\prime}, z\right) \wedge\right.$ $\varphi(z)) \leftrightarrow \chi_{0}\left(\bar{b}^{\prime}\right)$ and $\vDash \chi\left(\bar{a}, \bar{b}^{\prime}, c^{\prime}\right) \wedge \varphi\left(c^{\prime}\right) \leftrightarrow \chi_{1}\left(\bar{b}^{\prime}, c^{\prime}\right)$. Clearly in $N$ we have $\vDash \forall \bar{y}\left(\exists z \chi_{1}(\bar{y}, z) \leftrightarrow \chi_{0}(\bar{y})\right)$. From the definition of $T^{*}$ since $\exists z(\chi(\bar{a}, \bar{b}, z) \wedge \varphi(z))$ is a theorem, we have $\vDash \chi_{0}(\bar{b})$ in $M_{0}$ whence there exists $c$ in $\varphi\left(M_{0}\right)$ such that $\vDash \chi_{1}(\bar{b}, c)$ in $M_{0}$. From the definition of $T^{*}$ again we have $\chi(\bar{a}, \bar{b}, c) \wedge \varphi(c)$ a theorem of $T^{*}$ as required.

Let $\varphi_{0}\left(x, \bar{a}^{0}\right)$ and $\varphi_{1}\left(x, \bar{a}^{1}\right)$ be two s.m. formulas in a model $M$ of $T$. We say that $\varphi_{0}\left(x, \bar{a}^{0}\right)$ and $\varphi_{1}\left(x, \bar{a}^{1}\right)$ are linked by the pair $\left\langle\chi\left(x_{0}, x_{1}, \bar{y}^{0}, \bar{y}^{1}\right), m\right\rangle$ if the following formulas are valid in $M$

$$
\chi\left(x_{0}, x_{1}, \bar{a}^{0}, \bar{a}^{1}\right) \longrightarrow \varphi_{0}\left(x_{0}, \bar{a}^{0}\right) \wedge \varphi_{1}\left(x_{1}, \bar{a}^{1}\right), \exists^{\leqq m} x_{1} \chi\left(x_{0}, x_{1}, \bar{a}^{0}, \bar{a}^{1}\right),
$$

and

$$
\exists^{\leqq m} x_{1} \forall x_{0}\left[\neg \chi\left(x_{0}, x_{1}, \bar{a}^{0}, \bar{a}^{1}\right)\right] .
$$


Let $\varphi(x, \bar{a})$ be an s.m. formula in a model $M$ of $T$ we say that all copies of $\varphi(x, \bar{a})$ are uniformly related if there exists a pair $\langle\chi, m\rangle$, such that for any model $N$ of $T$, and linked copies $\varphi\left(x, \bar{a}^{0}\right)$, $\varphi\left(x, \bar{a}^{1}\right)$ of $\varphi(x, \bar{a})$ in $N$ are linked by $\langle\chi, m\rangle$.

LEMMA 10. Let $\varphi(x, \bar{a})$ be an s.m. formula in a model of an $\omega$-stable theory $T$. Suppose that all copies of $\varphi(x, \bar{a})$ are uniformly related. If $\varphi\left(x, \bar{a}^{0}\right)$ and $\varphi\left(x, \bar{a}^{1}\right)$ are two linked copies of $\varphi(x, \bar{a})$ in a model of $T$, then they have the same dimension.

Proof. For proof by contradiction suppose $M$ is a model of $T$ in which $\varphi\left(x, \bar{a}^{0}\right)$ and $\varphi\left(x, \bar{a}^{1}\right)$ are linked copies of $\varphi(x, \bar{a})$ with different dimensions. Notice that both must have finite dimensions: if both were infinite then we should have

$$
\operatorname{dim}\left(\varphi\left(x, \bar{a}^{0}\right), M\right)=\left|\varphi\left(M, \bar{a}^{0}\right)\right|=\left|\varphi\left(M, \bar{a}^{1}\right)\right|=\operatorname{dim}\left(\varphi\left(x, \bar{a}^{1}\right), M\right),
$$

while if one were finite and the other infinite we would have an infinite indiscernible set contained in the closure of a finite set which would contradict the superstability of $T$. Without loss of generality suppose that $\operatorname{dim}\left(\varphi\left(x, \bar{a}^{0}\right), M\right)=0$ and that $\operatorname{dim}\left(\varphi\left(x, \bar{\alpha}^{1}\right), M\right)=1$.

Using Lemma 9 we can form a model which we shall also call $M$ in which there are $\omega$ pairwise linked copies $\varphi\left(x, \bar{a}^{0}\right), \varphi\left(x, \bar{a}^{1}\right), \ldots$ of $\varphi(x, \bar{a})$ such that $\operatorname{dim}\left(\varphi\left(x, \bar{a}^{i}\right), M\right)=i$ and such that for any $i$ and $j, \bar{a}^{0 \cap} \bar{a}^{1 \cap} \ldots \cap \bar{a}^{j}$ and $\bar{a}^{i \cap} \bar{a}^{i+1 \cap} \ldots{ }^{n} \bar{a}^{i+j}$ realize the same type. Without loss we shall suppose that for any $i, j \varphi\left(M, \bar{a}^{i}\right) \cap \varphi\left(M, \bar{a}^{j}\right)=\varnothing$ and that $\varphi\left(M, \bar{a}^{i}\right)$ and $\varphi\left(M, \bar{a}^{j}\right)$ are linked by $\langle\chi, m\rangle$.

If $c \in \varphi\left(M, \bar{a}^{i}\right), c^{\prime} \in \varphi\left(M, \bar{a}^{j}\right)$, and $i<j$ we say that $c$ and $c^{\prime}$ are $\chi$-related written $c \chi c^{\prime}$ if $\vDash \chi\left(c, c^{\prime}, \bar{a}^{i}, \bar{a}^{j}\right)$. Likewise if $\bar{c}, \bar{c}^{\prime}$ are sequences in $\varphi\left(M, \bar{a}^{i}\right)$ and $\varphi\left(M, \bar{a}^{j}\right)$ respectively and $i<j$ we say $\bar{c}$ is $\chi$-related to $\bar{c}^{\prime}$, written $\bar{c} \chi \bar{c}^{\prime}$, if corresponding members of the two sequences are $\chi$-related. Let $\bar{c}, \bar{c}^{\prime}$ be sequences we write $\bar{c} \kappa \bar{c}^{\prime}$ if there exist $i, j$ and sequences $\bar{e}^{i}, \bar{e}^{j}$ independent in $\varphi\left(M, \bar{a}^{i}\right), \varphi\left(M, \bar{a}^{j}\right)$ respectively such that $i<j, \bar{c} \chi \bar{e}^{i}, \bar{c} \bar{c}^{\prime} \bar{e}^{j}$, and $\bar{e}^{i} \chi \bar{e}^{j}$. Our immediate goal is to prove:

Proposition. Let $\pi\left(x_{0}, \cdots, x_{n-1}, y_{0}, \cdots, y_{n-1}\right)$ be a formula containing at most parameters from $\bar{a}^{0}$ and the free variables indicated such that, if $\bar{c}^{n} \bar{c}^{\prime}$ is an independent sequence of length $2 n$ in $\varphi\left(M, \bar{a}^{0}\right)$ with $\operatorname{lh} \bar{c}=\operatorname{lh} \bar{c}^{\prime}=n$, then $\vDash \pi\left(\bar{c}, \bar{c}^{\prime}\right)$. Then $\bar{c}, \bar{c}^{\prime}$ can be found in $\varphi\left(M, \bar{a}_{0}\right)$ such that $\vDash \pi\left(\bar{c}, \bar{c}^{\prime}\right)$ and $\bar{c} \kappa \bar{c}^{\prime}$.

Towards establishing the proposition we make two observations. Firstly, if $i<j$ and $\bar{e}^{i}$ is independent in $\varphi\left(M, \bar{a}^{i}\right)$ then there exists 
$\bar{e}^{j}$ in $\varphi\left(M, \bar{a}^{j}\right)$ such that $\bar{e}^{i} \chi \bar{e}^{j}$ and any such $\bar{e}^{j} i$ s independent. The reason for this is that $\bar{a}^{i \cap} \bar{a}^{j}$ realizes the same type as $\bar{a}^{0 n} \bar{a}^{j-i}$. If we were to adjoin in turn basis elements $e_{0}^{0}, \cdots, e_{n-1}^{0}$ to $\varphi\left(M, \bar{a}^{0}\right)$ there would simultaneously be generated in $\varphi\left(M, \bar{a}^{j-i}\right)$ elements $e_{0}^{j-i}, \cdots, e_{n-1}^{j-i} \chi$-related to $e_{0}^{0}, \cdots, e_{n-1}^{0}$, respectively. Further $\bar{e}^{j-i}=$ $\left\langle e_{0}^{j-i}, \cdots, e_{n-1}^{j-i}\right\rangle$ is independent because no term is in the algebraic closure of those preceding it. Secondly, notice that there exists $k<\omega$ such that for every $i$ at most $k$ members of $\varphi\left(M, \bar{a}^{i}\right)$ have no $\chi$-predecessors in $\varphi\left(M, \bar{a}^{0}\right)$.

To prove the Proposition consider a fixed formula $\pi(\bar{x}, \bar{y})$ satisfying the hypothesis for which the conclusion fails. Let $I \subset \omega$ be infinite and $m<\omega$ be given. From the remarks in the last paragraph we can deduce the following. There exists $i \in I$, an infinite set $J \subset I$, an independent $m$-tuple $\bar{e}(m)$ in $\varphi\left(M, \bar{a}^{i}\right)$, and an $m$-tuple $\bar{c}(m)$ in $\varphi\left(M, \bar{a}^{0}\right)$ such that for every $j \in J$ there exists an independent $m$-tuple $\bar{e}^{j}(m)$ in $\varphi\left(M, \bar{a}^{j}\right)$, and an $m$-tuple $\bar{c}^{j}(m)$ in $\varphi\left(M, \bar{a}^{0}\right)$ such that

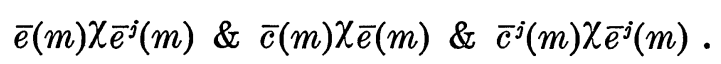

Further $\bar{c}(m)$ may be chosen such that if $\bar{c}$ is any $n$-tuple of distinct members of $\bar{c}(m)$, and $\bar{d}$ is an $n$-tuple in $\varphi\left(x, \bar{a}^{0}\right)$ independent over Rng $\bar{c}$, then $\vDash \pi(\bar{c}, \bar{d})$. To see this, in the first instance choose $\bar{c}(m)$ and $\bar{e}(m)$ of length much greater than $m$. There is a formula $\pi^{\prime}(\bar{x})$ equivalent to

$$
\exists^{\geq \omega} y_{0} \exists^{\geqq \omega} y_{1} \ldots \exists^{\geq \omega} y_{n-1}\left(\pi(\bar{x}, \bar{y}) \& \varphi\left(y_{0}, \bar{a}^{0}\right) \& \cdots \& \varphi\left(y_{n-1}, \bar{a}^{0}\right)\right)
$$

for arguments in $\varphi\left(M, \bar{a}^{0}\right)$ because in any family of finite subsets of s.m. sets which are uniformly definable there is a member of greatest cardinality. Our aim can be achieved by thinning $\bar{c}(m)$ to an $m$-tuple such that for any $n$-tuple $\bar{c}$ of distinct members of $\bar{c}(m)$ we have $\vDash \pi^{\prime}(\bar{c})$. Since we are supposing that the conclusion of the Proposition fails for $\pi(\bar{x}, \bar{y})$ if $\bar{c}$ is an $n$-tuple of distinct members of $\bar{c}(m)$ and $\bar{c}^{\prime}$ is the corresponding $n$-tuple of members of $\bar{c}^{\prime}(\mathrm{m})$ then $\vDash \neg \pi(\bar{c}, \bar{c})$.

Using the compactness theorem we can find $M^{\omega} \geqq M$, sequences $\left\langle\bar{c}(m): m\langle\omega\rangle,\left\langle\bar{c}^{\omega}(m): m\langle\omega\rangle,\left\langle i(m): m\langle\omega\rangle,\left\langle\bar{e}(m): m\langle\omega\rangle,\left\langle\bar{e}^{\omega}(m): m<\right.\right.\right.\right.\right.$ $\omega\rangle$, and $\bar{a}^{\omega} \in M^{\omega}$ such that the following conditions are satisfied. Firstly $\bar{a}^{\omega}$ realizes the same type as $\bar{a}$. For each $m, \bar{c}(m)$ and $\bar{c}^{\omega}(m)$ are $m$-tuples in $\varphi\left(M, \bar{a}^{0}\right)$ and $\varphi\left(M^{\omega}, \bar{a}^{0}\right)$ respectively, $i(m)<\omega, \bar{e}(m)$ is an independent $m$-tuple in $\varphi\left(M, \bar{a}^{i(m)}\right)$ and $\bar{e}^{\omega}(m)$ is an independent $m$-tuple in $\varphi\left(M^{\omega}, \bar{a}^{\omega}\right)$. Also, if $\bar{c}$ is any $n$-tuple of distinct members of $\bar{c}(m)$ then $\vDash \pi^{\prime}(\bar{c})$, and if $\bar{c}^{\omega}$ is the corresponding $n$-tuple of members of $\bar{c}^{\omega}(m)$ then $\vDash \neg \pi\left(\bar{c}, \bar{c}^{\prime}\right)$. Finally,

$$
\bar{e}(m) \chi_{\bar{e}^{\omega}}(m) \& \bar{c}(m) \chi \bar{e}(m) \& \bar{c}^{\omega}(m) \chi_{\bar{e}^{\omega}(m)}
$$


where $\chi$-relatedness is extended to the copy $\varphi\left(x, \bar{a}^{\omega}\right)$ of $\varphi(x, \bar{a})$ in the obvious way.

For each $m$ let $\bar{d}^{\omega}(m)$ be a subsequence of $\bar{c}^{\omega}(m)$ chosen such that $\bar{d}^{\omega}(m)$ is independent over $\bar{a}^{\omega}$ in $\varphi\left(M, \bar{a}^{0}\right)$, and such that $\bar{c}^{\omega}(m)$ is in cl (Rng $\left.\left(\bar{d}^{\omega}(m)^{n} \bar{a}^{\omega \cap} \bar{a}_{0}\right)\right)$. Now $\ln \left(\bar{d}^{\omega}(m)\right)<n$ because if $\bar{c}^{\omega}$ is a subsequence of $\bar{c}^{\omega}(m)$ of length $n$ and $\bar{c}$ is the corresponding subsequence of $\bar{c}(m)$ then $\vDash \neg \pi\left(\bar{c}, \bar{c}^{\omega}\right)$ and $\vDash \pi^{\prime}(\bar{c})$ whence $\bar{c}^{\omega}$ is not independent. Notice that $\bar{e}^{\omega}(m)$ is in $\operatorname{cl}\left(\bar{c}^{\omega}(m)^{\cap} \bar{a}^{\omega} \cap \bar{a}^{0}\right)$ and recall that $\bar{e}^{\omega}(m)$ is an independent sequence of length $m$ in $\varphi\left(M^{\omega}, \bar{a}^{\omega}\right)$. Let $\bar{d}^{\omega}$ be a sequence in $\varphi\left(M^{\omega}, \bar{a}^{0}\right)$ which is independent over $\bar{a}^{\omega}$ and which has maximal length $<n$. Comparing $\bar{d}^{\omega}$ and $\bar{d}^{\omega}(m)$ we see that $\operatorname{cl}\left(\bar{d}^{\omega \cap} \bar{a}^{\omega n} \bar{a}^{0}\right) \cap$ $\varphi\left(M^{\omega}, \bar{a}^{\omega}\right)$ has dimension at least $m$. Hence $\operatorname{cl}\left(\bar{d}^{\omega \cap} \bar{a}^{\omega \cap} \bar{a}^{0}\right) \cap \varphi\left(M^{\omega}, \bar{a}^{\omega}\right)$ has infinite dimension. This is inconsistent with $T$ being superstable. This completes the proof of the Proposition.

From the Proposition by compactness we can obtain $M^{\prime} \geqq M \bar{a}^{\prime}$ and $\bar{a}^{\prime \prime}$ in $M^{\prime}$ realizing the same type as $\bar{a}$, and an independent sequence $\left\langle c_{i}: i\langle\omega\rangle^{\cap}\left\langle c_{j}^{\prime}: i\langle\omega\rangle\right.\right.$ in $\varphi\left(M^{\prime}, \bar{a}^{0}\right)$ with the following property. There exist sequences $\left\langle e_{i}^{\prime}: i\langle\omega\rangle\right.$ and $\left(e_{i}^{\prime \prime}: i\langle\omega\rangle\right.$ in $\varphi\left(M, \bar{a}^{\prime}\right)$ and $\varphi\left(M, \bar{a}^{\prime \prime}\right)$ respectively such that $\left\langle c_{i}: i\langle\omega\rangle\right.$ is $\chi$-related to $\left\langle e_{i}^{\prime}: i\langle\omega\rangle,\left\langle e_{i}^{\prime}: i\langle\omega)\right.\right.$ is $\chi$-related to $\left\langle e_{i}^{\prime \prime}: i\langle\omega\rangle\right.$ and $\left\langle c_{i}^{\prime}: i\langle\omega\rangle\right.$ is $\chi$-related to $\left\langle e_{i}^{\prime \prime}: i\langle\omega\rangle\right.$. Again the notion of $\chi$-relatedness is extended to the present context in the obvious way. Let $\bar{a}^{0}$ and $\left\langle c_{i}: i\langle\omega\rangle\right.$ be fixed. We describe the relationship between $\bar{a}^{\prime}, \bar{a}^{\prime \prime}$ and $\left\langle c_{i}^{\prime}: i\langle\omega\rangle\right.$ by saying that $\bar{a}^{\prime \cap} \bar{a}^{\prime \prime}$ permits $\left\langle c_{i}^{\prime}: i\langle\omega\rangle\right.$.

Given two copies of $\varphi(x, \bar{a})$ and a solution $c$ of one there are at most a finite number of $c^{\prime}$ in the second copy to which $c$ is $\chi$-related. Also for all but a finite number of $c^{\prime}$ in the second copy there are only a finite number of $c$ in the first which are $\chi$-related to $c^{\prime}$. Thus if $|C| \leqq \lambda, \bar{b}^{\cap} \bar{b}^{\prime}$ permits at most $2^{\omega}+\lambda$ sequences in $C$. Choose $\lambda$ such that $\lambda^{\omega}>\lambda \geqq 2^{\omega}$ and $M^{\prime}$ to be $\lambda^{+}$-saturated. Choose $C \leqq \varphi\left(M, \bar{b}^{0}\right)$ such that $|C|=\lambda$ and $C$ is independent over $\left\langle c_{i}: i\langle\omega\rangle\right.$. There are $\lambda^{\omega}$ sequences $\left\langle c_{i}^{\prime}: i\langle\omega\rangle\right.$ in $C$ which have no repetitions and which thus make $\left\langle c_{i}: i\langle\omega\rangle^{n}\left\langle c_{i}^{\prime}: i\langle\omega\rangle\right.\right.$ independent. Each one is permitted by some $\bar{b}^{\cap} \bar{b}^{\prime}$ and at most $\lambda$ are permitted by the same $\bar{b}^{n} \bar{b}^{\prime}$. Hence over (Rng $\left.\bar{b}^{0}\right) \cup\left\{c_{i}: i<\omega\right\} \cup C$ there are at least $\lambda^{\omega}$ types realized by $2 n$-tuples $\bar{b}^{\cap} \bar{b}^{\prime}$ in $M^{\prime}$. This contradicts the superstability of $T$ and completes the proof of the lemma.

\section{The main result. We prove:}

THEOREM. Let $T$ be countable, $\omega$-stable, and neither $\omega$-categorical nor $\omega_{1}$-categorical. Then $T$ has $\geqq \omega$ models in every infinite power. 
Proof. As in [3] we work within a highly saturated model $\bar{M}$ of $T$. Let $\varphi(x, \bar{a})$ be an s.m. formula. Note that it may be impossible to choose $\bar{a}$ in the prime model. The proof splits into two cases according as $\varphi(x, \bar{a})$ is $\omega$-categorical or not. In the former case we modify the proof given in [3]. In the latter case we can distinguish enough different models by examining the dimensions of various copies of $\varphi(x, \bar{a})$.

Until further notice let $\varphi(x, \bar{a})$ be $\omega$-categorical. Let $\bar{a}$ realize an $l$-type $p_{0}$. Since $T$ is not $\omega$-categorical assume without loss of generality that $T$ has infinitely many 1-types. Choose $\psi(x)$ of least rank and degree in the sense of Morley such that $\psi(x)$ belongs to infinitely many distinct 1-types. By an observation of Shelah $\psi(x)$ can be chosen such that: there is a formula $\varepsilon(x, y)$ defining an equivalence relation on $\psi(\bar{M}), \psi(x)$ is equivalent to $\exists y \varepsilon(x, y)$, if $\vDash \psi(a)$ then $\varepsilon(x, \alpha)$ has rank $\alpha$ and degree 1 where $\alpha$ denotes the rank of $\psi(x)$, and the equivalence classes are indistinguishable in the sense that for any formula $\chi(x)$

$$
\vDash \forall x \forall y(\exists z(\varepsilon(x, z) \wedge \chi(z)) \wedge \varepsilon(y, y) \longrightarrow \exists w(\varepsilon(y, w) \wedge \chi(w))) .
$$

Fix an infinite power $\kappa$ and $n<w$. We now describe a model $M(\kappa, n)$ of $T$ of power $\kappa$. Let $\bar{a}(\kappa, n)=\left(a_{0}(\kappa, n), \cdots, a_{l}(\kappa, n)\right)$ realize $p_{0}$. Without loss of generality we may suppose that $\psi\left(x_{0}\right) \in p_{0}$. Let $I(\kappa, n)=\left\{b_{0}(\kappa, n), \cdots, b_{n-1}(\kappa, n)\right\}$ be such that for all $a \in I(\kappa, n)$, $\vDash \varepsilon\left(a, a_{0}(\kappa, n)\right)$ and $a$ realizes a type of $\operatorname{rank} \alpha$ over $(I(\kappa, n)-\{a\}) \cup$ Rng $\bar{a}(\kappa, n)$. Further let $J(\kappa, n)=\left\{c_{i}(\kappa, n): i<\kappa\right\}$ be a set of power $\kappa$ such that for any $a \in J(\kappa, n), \vDash \varphi(a, \bar{a}(\kappa, n))$ and the type realized by $a$ over Rng $a(\kappa, n) \cup I(\kappa, n) \cup(J(\kappa, n)-\{a\})$ is nonalgebraic. $M(\kappa, n)$ is to be a model prime over Rng $\bar{a}(\kappa, n) \cup I(\kappa, n) \cup J(\kappa, n)$.

Observe that $I(\kappa, n)$ is a subset of the solution set of $\varepsilon\left(x, a_{0}(\kappa, n)\right)$, a formula of rank $\alpha$ and degree 1 , whence $I(\kappa, n)$ is indiscernible over Rng $\bar{a}(\kappa, n)$. To see that in fact $I(\kappa, n)$ can be suitably chosen note that we can find $b_{i}(\kappa, n)$ such that for each $i<\omega \vDash \varepsilon\left(b_{i}(\kappa, n)\right.$, $a_{0}(\kappa)$ ) and $b_{i}(\kappa, n)$ realizes a type of rank $\alpha$ over $\left\{b_{j}(\kappa, n): j<i\right\} \cup$ $\cup$ Rng $\bar{a}(\kappa, n)$. Because for each $i$ there is only one such type containing $\varepsilon\left(x, a_{0}(\kappa, n)\right)$ the sequence $\left\langle b_{i}(\kappa, n): i\langle\omega\rangle\right.$ is indiscernible over Rng $\bar{a}(\kappa, n)$. Since the theory we are dealing with is stable, the set $\left\{b_{i}(\kappa, n): i<\omega\right\}$ is indiscernible. Similarly we see that $J(\kappa, n)$ can be found, that $J(\kappa, n)$ is indiscernible over $I(\kappa, n) \cup \operatorname{Rng}(\kappa, n)$ and that the type over Rng $\bar{a}(\kappa, n)$ realized by a sequence of distinct members of $I(\kappa, n) \cup J(\kappa, n)$ depends only upon which members of the sequence fall into $I(\kappa, n)$ and which into $J(\kappa, n)$. The following should be evident:

Proposition 1. If $m>n$ then the type realized by 


$$
\bar{a}(\kappa, m)^{\cap}\left\langle b_{i}(\kappa, m): i\langle n\rangle^{\cap}\left\langle c_{i}(\kappa, m): i\langle\kappa\rangle\right.\right.
$$

is the same as that realized by

$$
\bar{a}(\kappa, n)^{\cap}\left\langle b_{i}(\kappa, n): i\langle n\rangle^{\cap}\left\langle c_{i}(\kappa, n): i\langle\kappa\rangle .\right.\right.
$$

We also have:

Proposition 2. If $p \in S_{m}(I(\kappa, n) \cup J(\kappa, n) \cup \operatorname{Rng} \bar{a}(\kappa, n))$ is isolated so is $p \mid(I(\kappa, n) \cup \operatorname{Rng} \bar{a}(\kappa, n))$.

To see this let $p$ be isolated over $I(\kappa, n) \cup \operatorname{Rn} a \bar{a}(\kappa, n) \cup J_{0}$ where $J_{0}=\left\{c_{0}, \cdots, c_{j-1}\right\}$ is a finite subset of $J$. Now $\varphi(x, \bar{a}(\kappa, n))$ is s.m. and $\omega$-categorical, and as observed earlier $\varphi(x, \bar{a}(\kappa, n))$ remains $\omega$ categorical if names for a finite number of elements are added to the language. It follows immediately that $\left\langle c_{0}, \cdots, c_{j-1}\right\rangle$ realizes an isolated $j$-type over $I(\kappa, n) \cup \operatorname{Rng} \bar{a}(\kappa, n)$ which suffices.

We shall now show that if $m>n$ then $M(\kappa, m)$ and $M(\kappa, n)$ are not isomorphic. For proof by contradiction suppose that $M(\kappa, m)$ is isomorphic to $M(\kappa, n)$ and that $m>n$. Then some extension in $S_{l+n+1}(I(\kappa, n) \cup \operatorname{Rng} \bar{a}(\kappa, n) \cup J(\kappa, n))$ of the type $q^{+}$realized by $\bar{a}(\kappa, m)^{\cap}\left\langle b_{0}(\kappa, m), \cdots, b_{n}(\kappa, m)\right\rangle$ over $\varnothing$ is isolated. By Proposition 2 some extension $q^{\prime}$ of $q^{+}$in $S_{l+n+1}(I(\kappa, n) \cup \operatorname{Rng} \bar{a}(\kappa, n))$ is isolated. Let $\pi(\bar{x}, \bar{y}, \bar{z})$ be a formula such that

$$
\pi\left(\bar{x}, y, \bar{a}(\kappa, n), b_{0}(\kappa, n), \cdots, b_{n-1}(\kappa, n)\right)
$$

generates $q^{\prime}$ where $y$ stands for $x_{l+n}$. Notice from Proposition 1 that $q$, the type realized by $\bar{a}(\kappa, n)^{n}\left\langle b_{0}(\kappa, n), \cdots, b_{n-1}(\kappa, n)\right\rangle$ is included in $q^{+}$and hence in $q^{\prime}$. Let $\operatorname{lh}\left(\bar{a}^{0}\right)=\operatorname{lh}\left(\bar{a}^{1}\right)=l, \operatorname{lh}\left(\bar{b}^{0}\right)=\operatorname{lh}\left(\bar{b}^{1}\right)=n, \bar{a}^{0 \cap} \bar{b}^{0}$ realize $q$, and $\vDash \pi\left(\bar{a}^{1}, \bar{b}^{1}, b^{1}, \bar{a}^{0}, \bar{b}^{0}\right)$. Without loss assume that $\vDash \varepsilon\left(a_{0}^{1}, a_{0}^{0}\right)$; otherwise we may replace $\pi(\bar{x}, y, \bar{z})$ by a similar formula which does have this additional property. For example, if $\psi(x)$ has degree 3, $\pi(\bar{x}, y, \bar{z})$ may be replaced by

$$
\exists \bar{z}^{0} \exists y^{0} \exists \bar{z}^{1} \exists y^{1}\left(\pi\left(\bar{x}, y, \bar{z}^{1}\right) \wedge \pi\left(\bar{z}^{1}, y^{1}, \bar{z}^{0}\right) \wedge \pi\left(\bar{z}^{0}, y_{0}, \bar{z}\right)\right) .
$$

One should bear in mind here the indistinguishability of the $\varepsilon$-equivalence classes.

The following claim will enable us to complete the first half of the proof of the theorem:

Proposition 3. There exist $\bar{a}^{0}, \bar{b}^{0}, b^{1}, \bar{a}^{1}, b^{1}, b^{2}, \cdots$ such that

(i) for all $i<\omega, \bar{a}^{i \cap} \bar{b}^{i}$ realizes $q, \operatorname{lh}\left(\bar{a}^{i}\right)=l, \operatorname{lh}\left(\bar{b}^{i}\right)=n$, and $\vDash \pi\left(\bar{a}^{i+1}, \bar{b}^{i+1}, b^{i+1}, \bar{a}^{i}, \bar{b}^{i}\right)$ and

(ii) for $0<i<\omega, b^{i}$ realizes a type of rank $\alpha$ over $\left\{b^{j}: 0<\right.$ $j<\omega \wedge j \neq i\}$. 
Notice from (i) that $a_{0}^{i+1}$ and $a_{0}^{i}$ are $\varepsilon$-equivalent since $\vDash \pi\left(\bar{a}^{i+1}\right.$, $\left.\bar{b}^{i+1}, b^{i+1}, \bar{a}^{i}, \bar{b}^{i}\right)$ and $\bar{a}^{i \cap} \bar{b}^{i}$ realizes $q$. Further since $\bar{a}^{i+1 \cap} \bar{b}^{i+1}\left\langle b^{i+1}\right\rangle$ realizes $q^{+}, a_{0}^{i+1}$ and $b^{i+1}$ are $\varepsilon$-equivalent. Thus from (i) all of $b^{1}, b^{2}, \ldots$ are $\varepsilon$-equivalent to $a_{0}^{0}$. Now $\varepsilon\left(x, a_{0}^{0}\right)$ has rank $\alpha$ and degree 1 whence (ii) may be restated by saying that $\left\langle b^{i}: 0<i<\omega\right\rangle$ satisfies a certain $\omega$-type: Hence by the compactness theorem it is sufficient for the proof of the proposition to show that any finite initial segment of the desired sequence can be found. Suppose we wish to find $\bar{a}^{0}, \bar{b}^{0}$, $b^{1}, \bar{a}^{1}, \bar{b}^{1}, b^{2}, \cdots, \bar{a}^{j}, \bar{b}^{j}$ then we proceed in reverse order. First choose $\bar{a}^{j}, \bar{b}^{j}, b^{j}$ realizing $q^{+}$then choose $\bar{a}^{j-1}, \bar{b}^{j-1}$ such that $\vDash \pi\left(\bar{a}^{j}, \bar{b}^{j}, b^{j}\right.$, $\left.\bar{a}^{j-1}, \bar{b}^{j-1}\right)$. Notice from the choice of $\pi$ that $\exists \bar{z} \pi(\bar{x}, y, \bar{z}) \in q^{+}$. Now choose $b^{j-1}$ to realize the unique type over Rng $\left(\bar{a}^{j-1} \cap \bar{b}^{j-1}\right) \cup\left\{b^{j}\right\}$ which has rank $\alpha$ and contains $\varepsilon\left(x, a_{0}^{j-1}\right)$. Then $\bar{a}^{j-1 \cap} \bar{b}^{j-1}\left\langle b^{j-1}\right\rangle$ realizes $q^{+}$. We can choose $\bar{a}^{j-2}, \bar{b}^{j-2}$ such that $\vDash \pi\left(\bar{a}^{j-1}, \bar{b}^{j-1}, b^{j-1}, \bar{a}^{j-2}, \bar{b}^{j-2}\right)$. Let $b^{j-2}$ realize the unique type over Rng $\left(\bar{a}^{j-2 n} \bar{b}^{j-2}\right) \cup\left\{b_{j}, b_{j-1}\right\}$ which has rank $\alpha$ and contains $\varepsilon\left(x, a_{0}^{j-2}\right)$. Again $\bar{a}^{j-2 \cap} \bar{b}^{j-2 \cap}\left\langle b^{j-2}\right\rangle$ realizes $q^{+}$, thus we may continue in the same manner. Certainly (i) will be satisfied and for $0<i \leqq j, b_{i}$ realizes a type over $\left\{b^{k}: i<k \leqq j\right\}$ which has rank $\alpha$. Further $b^{1}, b^{2}, \cdots, b^{j}$ are all $\varepsilon$-equivalent. By the same argument used to show the existence of $I(\kappa, n)$, for $0<i \leqq j, b^{i}$ realizes a type of $\operatorname{rank} \alpha$ over $\left\{b^{k}: k \leqq j \wedge k \neq i\right\}$. This suffices to prove the proposition.

Of course, from (i) all of $b^{1}, b^{2}, \cdots$ are solutions of $\psi(x)$ and moreover are $\varepsilon$-equivalent. From (ii) we get that $\left\{b^{i}: 0<i<\omega\right\}$ is indiscernible. It is clear that the type realized by $\bar{a}^{i+1 n} \bar{b}^{i+1}\left\langle b^{i+1}\right\rangle$ over Rng $\left(\bar{a}^{i \cap b^{i}}\right)$ is isolated. From this it follows easily that for all $i, b^{i+1}$ realizes an isolated type over $\operatorname{Rng}\left(\bar{a}^{0 \cap} \bar{b}^{0}\right)$. Recall that $\psi(x)$ was chosen to have least possible rank and then least degree subject to there being infinitely many 1-types containing $\psi(x)$. Since the type of $b^{i+1}$ over $\varnothing$ has rank $\alpha$ it cannot be isolated. From Lemma 2 it follows that $b^{i+1}$ realizes a type of rank $<\alpha$ over $\operatorname{Rng}\left(\bar{a}^{0 \cap} \bar{b}^{0}\right)$.

Now choose $b^{i}$ for each $i, \omega \leqq i<2 \omega$, such that $\vDash \varepsilon\left(b^{i}, b^{1}\right)$ and $b^{i}$ realizes a type of rank $\alpha$ over Rng $\left(\bar{a}^{0 \cap} \bar{b}^{0}\right) \cup\left\{b^{j}: 0<j<i\right\}$. Using once again the argument by which we showed $I(n, \kappa)$ exists, we see that $\left\{b^{j}: 0<j<2 \omega\right\}$ is indiscernible. But $\bar{a}^{0 \cap} \bar{b}^{0}$ determines a partition of $\left\{b^{j}: 0<j<2 \omega\right\}$ into two infinite sets, since $b^{\omega}, b^{\omega+1}, \cdots$ are all the members of $\left\{b^{j}: 0<j<2 \omega\right\} \operatorname{Rng}\left(\bar{a}^{0}, \bar{b}^{0}\right)$ realizing a type of rank $\alpha$ over. Thus there are $2^{\omega}(l+n)$-types over the set $\left\{b^{j}: 0<j<2 \omega\right\}$ of power $\omega$. This contradicts the $\omega$-stability of $T$. Hence $M(\kappa, m)$ and $M(\kappa, n)$ are not isomorphic. This completes the proof of the theorem in the case where $T$ has an $\omega$-categorical s.m. formula.

For the rest of the proof suppose $\varphi(x, \bar{a})$ is an s.m. formula and that in any model $M$ containing $\bar{a}, \operatorname{cl}(\operatorname{Rng} \bar{a}) \cap \varphi(M, \bar{a})$ is infinite. Let $\bar{a}$ realize $p_{0}$. Suppose first of all that: 
(A) For each $n<\omega$ there exists a model $M$ of $T$ and copies $\varphi\left(x, \bar{a}_{0}\right), \cdots, \varphi\left(x, \bar{a}^{n-1}\right)$ of $\varphi(x, \bar{a})$ in $M$ no pair of which are linked.

With an arbitrary model $M$ of $T$ we associate a natural number $i(M)$ as follows. Let $E^{M}$ be the equivalence relation on $B=\{\bar{a}: \bar{a} \in M$ and $\bar{a}$ realizes $\left.p_{0}\right\}$ defined by: $\bar{a}^{0} E^{M} \widetilde{a}^{1}$ if $\varphi\left(x, \bar{a}^{0}\right)$ and $\varphi\left(x, \bar{a}^{1}\right)$ are linked. Recall that if two copies of $\varphi(x, \bar{a})$ are linked then they have the same dimension in $M$ modulo $\omega$. Let $i(M)$ be the number of equivalence classes $C$ under $E^{M}$ such that for $\bar{a} \in C \varphi(x, \bar{a})$ has dimension equal to $\|M\|$. If $B=\varnothing$ let $i(M)=0$. From Lemma 7 , for each $\kappa>\omega$ and each $n, 1 \leqq n<\omega$, there is a model $M$ of power $\kappa$ such that $i(M)=n$. When $\kappa=\omega$ the proof of Lemma 7 can be modified to show that there is a model $M$ of power $\omega$ with $i(M)=n$. Alternatively, from [3] $T$ has at least $\aleph_{0}$ countable models. In either case we have the desired conclusion. Henceforth we assume that (A) fails.

Let $\varphi\left(x, \bar{a}^{0}\right)$ and $\varphi\left(x, \bar{a}^{1}\right)$ be two copies of $\varphi(x, \bar{a})$ in a model $M$ of T. Recall that $\varphi\left(x, \bar{a}^{0}\right)$ and $\varphi\left(x, \bar{a}^{1}\right)$ are linked by the pair $\left\langle\chi\left(x^{0}, x_{1}, \bar{y}^{0}, \bar{y}^{1}\right), m\right\rangle$ if the following formulas are valid

$$
\begin{gathered}
\chi\left(x_{0}, x_{1}, \bar{a}^{0}, \bar{a}^{1}\right) \rightarrow \\
\varphi\left(x_{0}, \bar{a}^{0}\right) \wedge \varphi\left(x_{1}, \bar{a}^{1}\right), \quad \exists^{>m} x_{1} \chi\left(x_{0}, x_{1}, \bar{a}^{0}, \bar{a}^{1}\right) \\
\exists^{\leqq m} x_{1} \forall x_{0}\left[\neg \chi\left(x_{0}, x_{1}, \bar{a}^{0}, \bar{a}^{1}\right)\right] .
\end{gathered}
$$

We shall now show that because (A) fails we have:

(B) There exists a pair $\left\langle\chi\left(x_{0}, x_{1}, \bar{y}^{0}, \bar{y}^{1}\right), m\right\rangle$ such that if $\varphi\left(x, \bar{a}^{0}\right)$ and $\varphi\left(x, \bar{a}^{1}\right)$ are linked copies of $\varphi(x, \bar{a})$ in a model of $T$, then they are linked by $\left\langle\chi\left(x_{0}, x_{1}, \bar{y}^{0}, \bar{y}^{1}\right), m\right\rangle$. That is, all copies of $\varphi(x, \bar{a})$ are uniformly related.

Let $\left\langle\left\langle\chi_{i}, m_{i}\right\rangle: i\langle\omega\rangle\right.$ be an enumeration of all pairs which might link two copies of $\varphi(x, \bar{a})$. Notice that it is sufficient to prove that there exists $j<\omega$ such that any two linked copies are linked by $\left\langle\chi_{i}, m_{i}\right\rangle$ for some $i \leqq j$. For proof by contradiction suppose no such $j$ exists. Then in any $\omega$-saturated model we can find a sequence $\left\langle\varphi\left(x, \bar{a}^{i}\right\rangle: i\langle\omega\rangle\right.$ of copies of $\varphi(x, \bar{a})$ such that for each $i, \varphi\left(x, \bar{a}^{0}\right)$ and $\varphi\left(x, \bar{a}^{i}\right)$ are linked but not by any pair $\left\langle\chi_{k}, m_{k}\right\rangle$ with $k \leqq i$. Observe that for each $i$ and $j$ there exists $i^{\prime}$ such that if $k \geqq$ $i^{\prime} \varphi\left(x, \bar{a}^{2}\right)$ and $\varphi\left(x, \bar{a}^{k}\right)$ are not linked by any pair with subscript $\leqq j$. By the compactness theorem (A) holds. This contradiction completes the proof of (B).

We have an s.m. formula $\varphi(x, \bar{a})$ which has infinitely many algebraic solutions. If $\varphi(x, \bar{a})$ does not have the Vaught property then by Lemma $4 T$ has an inessential extension which is $\omega_{1}$-categorical. However, any theory which has an $\omega_{1}$-categorical inessential extension is itself $\omega_{1}$-categorical; one can see this from the remark at the end of $\S 2$ in [1]. Thus we may suppose that $\varphi(x, \bar{a})$ has the 
Vaught property. Let $\kappa>\omega$ be given. From Lemma $3 T$ has a model $M$ of power $\kappa$ containing $\bar{a}$ such that $\varphi(x, \bar{a})$ has finite dimension in $M$. Define $j(M)$ to be the greatest $n<\omega$ such that some copy of $\varphi(x, \bar{a})$ in $M$ has dimension $n$ and to be undefined if there are no copies of finite dimension. From Lemma 10 any linked copies of $\varphi(x, \bar{a})$ in $M$ have the same dimension, and since (A) fails the number of pairwise unlinked copies of $\varphi(x, \bar{a})$ in a model is finite. Thus $j(M)$ is well defined whenever there is some copy with finite dimension. Let $\left\{e_{0}, \cdots, e_{m}\right\}$ be a set of solutions of $\varphi(x, \bar{a})$ independent over $|M|$, and let $M^{\prime}$ be prime over $|M| \cup\left\{e_{0}, \cdots, e_{m}\right\}$. It is easy to see that $\varphi(x, \bar{a})$ has finite dimension $>m$ in $M^{\prime}$. Hence $j\left(M^{\prime}\right)>m$ and $\left|M^{\prime}\right|=\kappa$. From this it is clear that there are infinitely many nonisomorphic models of power $\kappa$. This completes the proof of the theorem.

4. Concluding remarks. As mentioned in the introduction our theorem together with a result of Shelah confirms the conjecture of W. K. Forrest that any countable theory having a finite number of models, but more than one, in an uncountable power is $\omega$-categorical. Forrest also conjectured that such theories have only a finite number of models in each power $\kappa, \boldsymbol{\aleph}_{0}<\kappa<\boldsymbol{\aleph}_{\omega}$. We have verified this. A question which is not so precise concerns the structure of such theories. In some sense every known example can be seen as a combination of a finite number of theories categorical in every infinite power. Can this idea be formalized and be demonstrated for all such theories?

\section{REFERENCES}

1. J. T. Baldwin and A. H. Lachlan, On strongly minimal sets, J. Symbolic Logic, 36 (1971), 79-96.

2. A. H. Lachlan, A property of stable theories, Fund. Math., 77 (1972), 9-20.

3. - On the number of countable models of a countable superstable theory, Logic Methodology and the Philosophy of Science IV, North Holland 1973, 45-56.

4. - Two conjectures regarding the stability of $\omega$-categorical theories, Fund. Math., 81 (1974), 133-145.

5. M. Morley, Categoricity in power, Trans. Amer. Math. Soc., 114 (1965), 514-518.

6. J. Rosenthal, A new proof of a theorem of Shelah, Proc. of the 1971 Symp. in Berkeley in honour of Tarski, published by Association for Symbolic Logic.

7. S. Shelah, Finite diagrams stable in power, Annals of Math. Logic, 2 (1970), 69118.

8. - Stability, the f.c.p., and superstability, model, theoretic properties of formulas in first-order theory, Annals of Math. Logic, 3 (1971), 271-362.

9. S. Shelah, forthcoming book on stability in first-order model theory.

Received January 16, 1975.

SimON FraSER UNIVERSITY 



\section{PACIFIC JOURNAL OF MATHEMATICS}

\section{EDITORS}

RICHARD ARENS (Managing Editor)

University of California

Los Angeles, California 90024

\author{
R. A. Beaumont \\ University of Washington \\ Seattle, Washington 98105
}

\section{J. DugundjI}

Department of Mathematics University of Southern California Los Angeles, California 90007

D. Gilbarg and J. Milgram

Stanford University

Stanford, California 94305

\section{ASSOCIATE EDITORS}
E. F. BECKENBACH
B. H. NeumanN
F. WOLF
K. YoshidA

\section{SUPPORTING INSTITUTIONS}

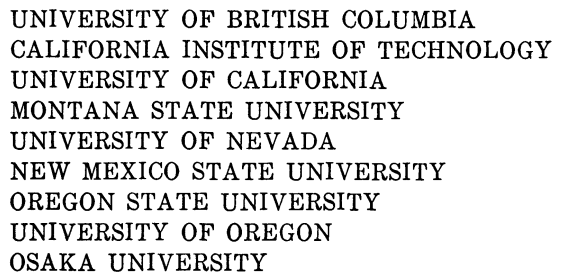

UNIVERSITY OF BRITISH COLUMBIA CALIFORNIA INSTITUTE OF TECHNOLOGY UNIVERSITY OF CALIFORNIA MONTANA STATE UNIVERSITY UNIVERSITY OF NEVADA NEW MEXICO STATE UNIVERSITY OSAKA UNIVERSITY OREGON STATE UNIVERSITY UNIVERSITY OF OREGON

\author{
UNIVERSITY OF SOUTHERN CALIFORNIA \\ STANFORD UNIVERSITY \\ UNIVERSITY OF TOKYO \\ UNIVERSITY OF UTAH \\ WASHINGTON STATE UNIVERSITY \\ UNIVERSITY OF WASHINGTON \\ AMERICAN MATHEMATICAL SOCIETY
}

The Supporting Institutions listed above contribute to the cost of publication of this Journal, but they are not owners or publishers and have no responsibility for its content or policies.

Mathematical papers intended for publication in the Pacific Journal of Mathematics should be in typed form or offset-reproduced, (not dittoed), double spaced with large margins. Please do not use built up fractions in the text of your manuscript. You may however, use them in the displayed equations. Underline Greek letters in red, German in green, and script in blue. The first paragraph or two must be capable of being used separately as a synopsis of the entire paper. Items of the bibliography should not be cited there unless absolutely necessary, in which case they must be identified by author and Journal, rather than by item number. Manuscripts, in triplicate, may be sent to any one of the editors. Please classify according to the scheme of Math. Reviews, Index to Vol. 39. All other communications should be addressed to the managing editor, or Elaine Barth, University of California, Los Angeles, California, 90024.

The Pacific Journal of Mathematics expects the author's institution to pay page charges, and reserves the right to delay publication for nonpayment of charges in case of financial emergency.

100 reprints are provided free for each article, only if page charges have been substantially paid. Additional copies may be obtained at cost in multiples of 50 .

The Pacific Journal of Mathematics is issued monthly as of January 1966. Regular subscription rate: $\$ 72.00$ a year $(6$ Vols., 12 issues). Special rate: $\$ 36.00$ a year to individual members of supporting institutions.

Subscriptions, orders for back numbers, and changes of address should be sent to Pacific Journal of Mathematics, 103 Highland Boulevard, Berkeley, California, 94708.

PUBLISHED BY PACIFIC JOURNAL OF MATHEMATICS, A NON-PROFIT CORPORATION

Printed at Kokusai Bunken Insatsusha (International Academic Printing Co., Ltd.), 8-8, 3-chome, Takadanobaba, Shinjuku-ku, Tokyo 160, Japan. 


\section{Pacific Journal of Mathematics}

\section{Vol. 61, No. 2 December, 1975}

Graham Donald Allen, Francis Joseph Narcowich and James Patrick Williams, An operator version of a theorem of Kolmogorov .......................

Joel Hilary Anderson and Ciprian Foias, Properties which normal operators share with normal derivations and related operators . . . . . . . . . . . . . . . . . . . . .

Constantin Gelu Apostol and Norberto Salinas, Nilpotent approximations and

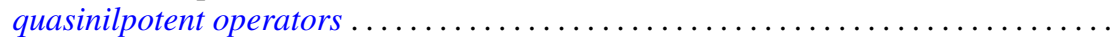

James M. Briggs, Jr., Finitely generated ideals in regular $F$-algebras . . . . . . . . . . .

Frank Benjamin Cannonito and Ronald Wallace Gatterdam, The word problem and power problem in 1-relator groups are primitive recursive ..................

Clifton Earle Corzatt, Permutation polynomials over the rational numbers ...........

L. S. Dube, An inversion of the $S_{2}$ transform for generalized functions . . . . . . . . . . William Richard Emerson, Averaging strongly subadditive set functions in unimodular

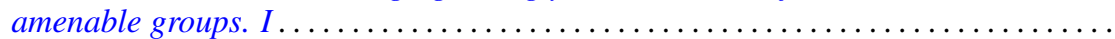

Barry J. Gardner, Semi-simple radical classes of algebras and attainability of

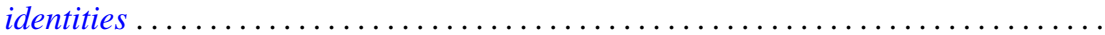

Irving Leonard Glicksberg, Removable discontinuities of A-holomorphic functions ....

Fred Halpern, Transfer theorems for topological structures . . . . . . . . . . . . . . .

H. B. Hamilton, T. E. Nordahl and Takayuki Tamura, Commutative cancellative

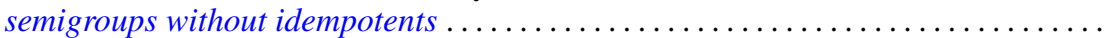

Melvin Hochster, An obstruction to lifting cyclic modules .....................

Alistair H. Lachlan, Theories with a finite number of models in an uncountable power

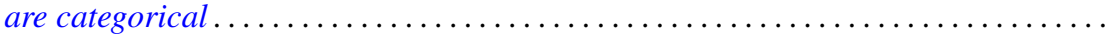

Kjeld Laursen, Continuity of linear maps from $C^{*}$-algebras . . . . . . . . . . . . .

Tsai Sheng Liu, Oscillation of even order differential equations with deviating arguments ....

Jorge Martinez, Doubling chains, singular elements and hyper- $Z$

Mehdi Radjabalipour and Heydar Radjavi, On the geometry of num Thomas I. Seidman, The solution of singular equations, I. Linear equations in Hilbert

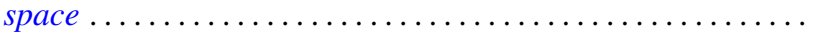

R. James Tomkins, Properties of martingale-like sequences ......

Alfons Van Daele, A Radon Nikodým theorem for weights on von Neumann

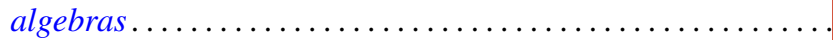

Kenneth S. Williams, On Euler's criterion for quintic nonresidues

Scott Andrew Wolpert, Noncompleteness of the Weil-Petersson metric for Teichmüller

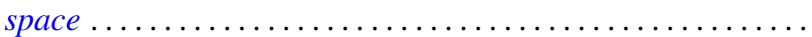

Volker Wrobel, Some generalizations of Schauder's theorem in locally convex

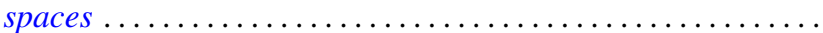

Kelly Denis McKennon, Corrections to: "Multipliers of type $(p, p)$ "; "Multipliers of type $(p, p)$ and multipliers of the group $L_{p}$-algebras"; "Multipliers and the

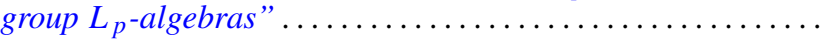

Andrew M. W. Glass, W. Charles (Wilbur) Holland Jr. and Stephen H. McCleary, Correction to: " $a *$-closures to completely distributive lattice-ordered

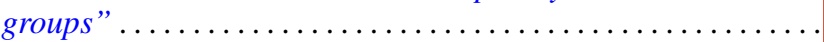

Zvi Arad and George Isaac Glauberman, Correction to: "A characteristic subgroup of

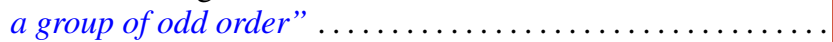

Roger W. Barnard and John Lawson Lewis, Correction to: "Subordination theorems

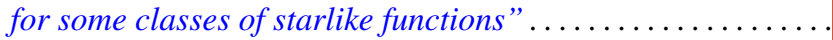

